\title{
ON A NONLINEAR ELLIPTIC SYSTEM WITH SYMMETRIC COUPLING
}

\author{
Oscar Agudelo \\ Departamento de Ingeniería Matemática \\ Universidad de Chile \\ Santiago, Chile \\ oagudel0@dim.uchile.cl
}

\author{
Bernhard Ruf \\ Dipartimento di Matematica \\ Università degli Studi di Milano \\ Milano, Italia \\ bernhard.ruf@unimi.it
}

\author{
Carlos Vélez \\ Escuela de Matemáticas \\ Universidad Nacional de Colombia, Sede Medellín \\ Medellín, Colombia \\ cauvelez@unal.edu.co
}

\begin{abstract}
Multiplicity results are proved for the nonlinear elliptic system

$$
\left\{\begin{array}{rll}
-\Delta u+g(v) & =0 & \\
-\Delta v+g(u) & =0 & \text { in } \Omega \\
u=v & =0 & \text { on } \partial \Omega
\end{array}\right.
$$

where $\Omega \subset \mathbb{R}^{N}$ is a bounded domain with smooth boundary and $g: \mathbb{R} \longrightarrow \mathbb{R}$ is a nonlinear $C^{1}$-function which satisfies addtional conditions. No assumption of symmetry on $g$ is imposed.

Extensive use is made of a global version of the Lyapunov-Schmidt reduction method due to Castro and Lazer (see $[\mathrm{C}]$ and [CL]), and of symmetric versions of the Mountain Pass Theorem (see $[\mathrm{AR}]$ and $[\mathrm{R}]$ ).
\end{abstract}

Key Words and phrases: Elliptic system, Lyapunov-Schmidt reduction method, Mountain Pass Theorem.

\section{Introduction}

It is well-known that a symmetry in a differential equation often generates the existence of multiple solutions. Consider e.g. the superlinear and subcritical equation

$$
-\Delta u=f(u), \text { in } \Omega,\left.u\right|_{\partial \Omega}=0
$$

where $f \in C(\mathbb{R})$ is a superlinear and subcritical nonlinearity. If $f(u)$ is an odd function, then the equation has the symmetry $u \mapsto-u$. Using the concept of index theories (e.g. the Krasnoselskii genus), one shows that this symmetry implies that the equation has infinitely many solutions.

\footnotetext{
${ }^{1}$ This work was supported by Progetto UniALA, offered by Fondazione Cariplo and Università degli Studi di Milano in 2010.
} 
In this article we consider a semilinear elliptic system in which the symmetry is not given by an odd nonlinearity, but by a symmetric coupling. We consider systems of the following form

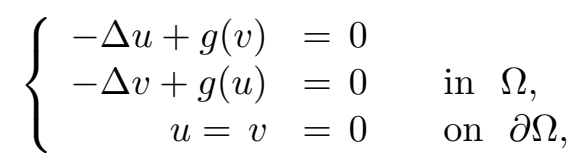

where $\Omega \subset \mathbb{R}^{N}, N \geq 2$, is a bounded domain with smooth boundary and $g: \mathbb{R} \longrightarrow \mathbb{R}$ is a $C^{1}$-function satisfying some assumptions to be specified later, but is not required to be odd. Note that this system allows the following symmetry:

$$
T_{1}:(u, v) \mapsto(v, u) .
$$

Indeed, looking at the associated functional (supposing it is well-defined)

$$
J(u, v)=\int_{\Omega} \nabla u \cdot \nabla v+\int_{\Omega} G(u)+\int_{\Omega} G(v),
$$

where $G(s)=\int_{0}^{s} g(t) d t$ is the primitive of $g$, we see that this functional is invariant under the group action $T=\left\{i d, T_{1}\right\}$.

Thus, one may try to proceed similarly as for equation (2) by defining a suitable index. However, one encounters two major problems. First, the functional is strongly indefinite due to the first term in the functional. Second, the group $T$ has an infinite-dimensional fixed point space, given by the pairs of functions of the form $\{(u, u)\}$. We overcome these difficulties by performing an infinite dimensional Lyapunov-Schmidt reduction (following Castro-Lazer [CL]). Surprisingly, the resulting reduced functional $\widetilde{J}$ has the classical $\mathbb{Z}_{2}$-symmetry $\{i d,-i d\}$ (although, as we emphasize, no oddness assumption is taken for the nonlinearity), and so classical variational methods for the existence of multiple solutions can be employed.

We will denote by $0<\lambda_{1}<\lambda_{2} \leq \cdots \leq \lambda_{k} \leq \cdots$ the sequence of eigenvalues of $-\Delta$ with zero Dirichlet boundary condition in $\Omega$. Also, $\left\{\varphi_{j}\right\}_{j}$ will denote an orthonormal basis, in $H_{0}^{1}(\Omega)$, of eigenfunctions of $-\Delta$ in $\Omega$ with Dirichlet boundary condition. We will study the existence of multiple solutions for problem (3) under three different sets of conditions. For the first two sets, we assume $g$ satisfies

$\left(g_{0}\right) g(0)=0$ and

$\left(g_{1}\right) \inf _{t \in \mathbb{R}} g^{\prime}(t)>-\lambda_{1}$.

First, we consider the superlinear setting, in which we assume

$\left(g_{2}\right)$ There exists a positive constant $C$ such that

$$
|g(t)| \leq C\left(1+|t|^{p}\right) \text {, where } p \in\left(1, \frac{N+2}{N-2}\right) \text { for all } t \in \mathbb{R} \text {, and }
$$

$\left(g_{3}\right)$ There exists $R>0$ such that $0<\mu G(t) \leq t g(t)$, for $|t|>R$, where $\mu>2$.

Secondly, we also consider the asymptotically linear setting, in which $g$ is assumed to satisfy

$$
\left(g_{4}\right) g^{\prime}(\infty):=\lim _{|t| \rightarrow \infty} \frac{g(t)}{t} \in\left(\lambda_{k}, \lambda_{k+1}\right) \text { for some } k \geq 1 \text {. }
$$


Our main results read as follows.

Theorem A. (superlinear case) If g satisfies $\left(g_{0}\right)-\left(g_{3}\right)$, problem (3) has infinitely many solutions.

We observe that conditions $\left(g_{2}\right)$ and $\left(g_{3}\right)$ include the "classical" nonlinearity $g(t)=$ $t|t|^{p-1}$. But we emphazise that Theorem A holds true for a more general kind of nonlinearities, e.g. $g(t)=\left(t^{+}\right)^{p}-\left(t^{-}\right)^{q}$, for $t \in \mathbb{R}$ and $1<p, q<(N+2) /(N-2)$, without any further restriction on $p$ and $q$.

In the asymptotically linear framework we have the following analogue of Theorem A.

Theorem B. (asymptotically linear case) Assume g satisfies $\left(g_{0}\right)-\left(g_{1}\right)$ and $\left(g_{4}\right)$. If, in addition, $g^{\prime}(0)<\lambda_{j}$ for $j \leq k$, then problem $(3)$ has (at least) $2(k-j+1)$ nontrivial solutions.

On the other hand, we consider a third setting, in which we only assume

$\left(g_{5}\right) \sup _{t \in \mathbb{R}} g^{\prime}(t)<\lambda_{1}$.

We observe that under condition $\left(g_{5}\right)$, system $(3)$ is equivalent to the system

$$
\left\{\begin{array}{rlrl}
-\Delta u & =h(v) & \\
-\Delta v & =h(u) & \text { in } \quad \Omega \\
u & =v=0 & & \text { on } \partial \Omega
\end{array}\right.
$$

where $h=-g$ satisfies inf $h^{\prime}>-\lambda_{1}$. We point out that (5) is the very analogue in systems of the single-equation problem (2). In this direction we prove the following result which shows that system (3) (or, equivalently, system (5)) has a strong hidden symmetry.

Theorem C. Assume $g$ satisfies $\left(g_{5}\right)$. Then $(u, v)$ is a solution of (3) if and only if $u \equiv v$ and

$$
-\Delta u+g(u)=0, \quad \text { in } \Omega,\left.u\right|_{\partial \Omega}=0 .
$$

In other words, under condition $\left(g_{5}\right)$, solving system (3) is equivalent to solving the single-equation problem (6).

System (3) is Hamiltonian and our approach to it is variational, i.e. we define an energy functional $J: H_{0}^{1}(\Omega) \times H_{0}^{1}(\Omega) \longrightarrow \mathbb{R}$ by

$$
J(u, v)=\int_{\Omega}(\nabla u \cdot \nabla v+G(u)+G(v)) d \zeta
$$

where $G(t):=\int_{0}^{t} g(s) d s$. Assuming either $\left(g_{2}\right)$ or $\left(g_{4}\right)$, this functional is of class $C^{1}$ (see $[\mathrm{R}]$ ) and

$$
\partial_{u} J(u, v) \varphi=\int_{\Omega}(\nabla \varphi \cdot \nabla v+g(u) \varphi) d \zeta, \quad \forall u, v, \varphi \in H_{0}^{1}(\Omega),
$$

and

$$
\partial_{v} J(u, v) \psi=\int_{\Omega}(\nabla u \cdot \nabla \psi+g(v) \psi) d \zeta, \quad \forall u, v, \psi \in H_{0}^{1}(\Omega)
$$


Thus, because of classical regularity theory (see [GT]), critical points of $J$ agree with classical solutions of problem (3). We then prove Theorem A and B showing the existence of critical points of $J$. Because of the form of the system

$$
(u, v) \text { is a solution of (3) if and only if }(v, u) \text { is a solution of (3), }
$$

as can be easily verified. This fact provides some symmetry on the functional $J$ when it is written in appropriate coordinates.

The paper is organized as follows: in Section 2 we recall the Castro-Lazer version of the Lyapunov-Schmidt reduction method in an abstract setting. We then show that our functional $J$ satisfies the conditions of such setting. In Section 3 we prove Theorem A and in Section 4 we prove Theorem B. In proving them, we recall and use appropriate symmetric versions of the Mountain Pass Theorem of Ambrosetti and Rabinowitz. Finally, in Section 5 we prove Theorem C.

\section{Preliminaries}

We begin by stating a global version of the Lyapunov-Schmidt method (see $[\mathrm{C}]$ and $[\mathrm{CL}])$.

Lemma 2.1. Let $H$ be a real separable Hilbert space. Let $Z$ and $W$ be closed subspaces of $H$ such that $H=Z \oplus W$. Let $J: H \longrightarrow \mathbb{R}$ a function of class $C^{1}$. If there exist $m>0$ and $\sigma>1$ such that

$$
\left\langle\nabla J(\boldsymbol{z}+\boldsymbol{w})-\nabla J\left(\boldsymbol{z}+\boldsymbol{w}_{1}\right), \boldsymbol{w}-\boldsymbol{w}_{1}\right\rangle \geq m\left\|\boldsymbol{w}-\boldsymbol{w}_{1}\right\|_{H}^{\sigma} \quad \forall \boldsymbol{z} \in Z \quad \forall \boldsymbol{w}, \boldsymbol{w}_{1} \in W
$$

then:

(i) There exists a continuous function $\phi: Z \rightarrow W$ such that

$$
J(\boldsymbol{z}+\phi(\boldsymbol{z}))=\min _{\boldsymbol{w} \in W} J(\boldsymbol{z}+\boldsymbol{w}) .
$$

Moreover, given $\boldsymbol{z} \in Z, \phi(\boldsymbol{z})$ is the unique element of $W$ such that

$$
\langle\nabla J(\boldsymbol{z}+\phi(\boldsymbol{z})), \boldsymbol{w}\rangle=0 \quad \forall \boldsymbol{w} \in W .
$$

(ii) The functional $\widetilde{J}: Z \rightarrow \mathbb{R}$, defined by $\widetilde{J}(\boldsymbol{z}):=J(\boldsymbol{z}+\phi(\boldsymbol{z}))$ for $\boldsymbol{z} \in Z$, is of class $C^{1}$. Moreover,

$$
D \widetilde{J}(\boldsymbol{z}) \boldsymbol{h}=\langle\nabla \widetilde{J}(\boldsymbol{z}), \boldsymbol{h}\rangle=\langle\nabla J(\boldsymbol{z}+\phi(\boldsymbol{z})), \boldsymbol{h}\rangle \quad \forall \boldsymbol{z}, \boldsymbol{h} \in Z .
$$

(iii) Given $\boldsymbol{z} \in Z, \boldsymbol{z}$ is a critical point of $\widetilde{J}$ if and only if $\boldsymbol{z}+\phi(\boldsymbol{z})$ is a critical point of $J$.

Assuming $\left(g_{1}\right)$ and either $\left(g_{2}\right)$ or $\left(g_{4}\right)$, we intend to apply Lemma 2.1 to the functional $J: H_{0}^{1}(\Omega) \times H_{0}^{1}(\Omega) \longrightarrow \mathbb{R}$ defined as

$$
J(u, v)=\int_{\Omega}(\nabla u \cdot \nabla v+G(u)+G(v)) d \zeta
$$


where $G(t):=\int_{0}^{t} g(s) d s$. First, it is well-known that assuming either $\left(g_{2}\right)$ or $\left(g_{4}\right)$, this functional is of the class $C^{1}$ (see $\left.[\mathrm{R}]\right)$ and

$$
\partial_{u} J(u, v) \varphi=\int_{\Omega}(\nabla \varphi \cdot \nabla v+g(u) \varphi) d \zeta, \quad \forall u, v, \varphi \in H_{0}^{1}(\Omega),
$$

and

$$
\partial_{v} J(u, v) \psi=\int_{\Omega}(\nabla u \cdot \nabla \psi+g(v) \psi) d \zeta, \quad \forall u, v, \psi \in H_{0}^{1}(\Omega) .
$$

Let us take $H=H_{0}^{1}(\Omega) \times H_{0}^{1}(\Omega)$ equipped with the inner product $\left\langle\left(u_{1}, v_{1}\right),\left(u_{2}, v_{2}\right)\right\rangle=$ $\left\langle u_{1}, u_{2}\right\rangle_{H_{0}^{1}}+\left\langle v_{1}, v_{2}\right\rangle_{H_{0}^{1}}$. Here, $\left\langle f_{1}, f_{2}\right\rangle_{H_{0}^{1}}=\int_{\Omega} \nabla f_{1} \cdot \nabla f_{2}$. Let us define $W:=$ $\left\{\mathbf{w}=(w, w): w \in H_{0}^{1}(\Omega)\right\}$ and $Z:=\left\{\mathbf{z}=(z,-z): z \in H_{0}^{1}(\Omega)\right\}$. Then $H_{0}^{1}(\Omega) \times H_{0}^{1}(\Omega)=Z \oplus W$. Let us verify (10). Let $\mathbf{z} \in Z$ and $\mathbf{w}, \mathbf{w}_{1} \in W$. Then

$$
\begin{aligned}
\left\langle\nabla J(\mathbf{z}+\mathbf{w})-\nabla J\left(\mathbf{z}+\mathbf{w}_{1}\right), \mathbf{w}-\mathbf{w}_{1}\right\rangle \\
=\left\langle\nabla J(z+w,-z+w)-\nabla J\left(z+w_{1},-z+w_{1}\right),\left(w-w_{1}, w-w_{1}\right)\right\rangle \\
=\left[\partial_{u} J(z+w,-z+w)-\partial_{u} J\left(z+w_{1},-z+w_{1}\right)\right]\left(w-w_{1}\right) \\
\quad+\left[\partial_{v} J(z+w,-z+w)-\partial_{v} J\left(z+w_{1},-z+w_{1}\right)\right]\left(w-w_{1}\right) \\
=2 \int_{\Omega}\left|\nabla\left(w-w_{1}\right)\right|^{2}+\int_{\Omega}\left[g(z+w)-g\left(z+w_{1}\right)\right]\left(w-w_{1}\right) \\
\quad+\int_{\Omega}\left[g(-z+w)-g\left(-z+w_{1}\right)\right]\left(w-w_{1}\right) .
\end{aligned}
$$

Because of $\left(g_{1}\right)$, there exists $\epsilon \in\left(0, \lambda_{1}\right)$ such that $g^{\prime}(t) \geq-\lambda_{1}+\epsilon$ for all $t \in \mathbb{R}$. Thus, the Mean Value Theorem, the previous identities, and Poincarï $i \frac{1}{2}$ 's Inequality give us

$$
\begin{aligned}
& \left\langle\nabla J(\mathbf{z}+\mathbf{w})-\nabla J\left(\mathbf{z}+\mathbf{w}_{1}\right), \mathbf{w}-\mathbf{w}_{1}\right\rangle \\
& \geq 2 \int_{\Omega}\left|\nabla\left(w-w_{1}\right)\right|^{2}+2\left(-\lambda_{1}+\epsilon\right) \int_{\Omega}\left(w-w_{1}\right)^{2} \\
& \geq 2 \int_{\Omega}\left|\nabla\left(w-w_{1}\right)\right|^{2}+2 \frac{\left(-\lambda_{1}+\epsilon\right)}{\lambda_{1}} \int_{\Omega}\left|\nabla\left(w-w_{1}\right)\right|^{2} \\
& =2 \frac{\epsilon}{\lambda_{1}} \int_{\Omega}\left|\nabla\left(w-w_{1}\right)\right|^{2}=\frac{\epsilon}{\lambda_{1}}\left\|\mathbf{w}-\mathbf{w}_{1}\right\|_{H}^{2} .
\end{aligned}
$$

We have then verified the hypotheses of Lemma 2.1. Thus, there exist a continuous function $\mathbf{w} \equiv \phi: Z \longrightarrow W$ and a functional $\widetilde{J}: Z \longrightarrow \mathbb{R}$ which satisfy (i), (ii) and (iii). Because of (iii), our concern becomes the existence of critical points of the functional $\widetilde{J}$.

Observe that, given $\mathbf{z}=(z,-z) \in Z, \mathbf{w}(\mathbf{z})=(w(z), w(z))$ and

$$
\begin{aligned}
\widetilde{J}(\mathbf{z}) & =J(z+w(z),-z+w(z)) \\
& =\int_{\Omega}\left[|\nabla w(z)|^{2}-|\nabla z|^{2}+G(z+w(z))+G(-z+w(z))\right] d \zeta .
\end{aligned}
$$

The symmetry of problem (3) expressed by condition (9) is translated into the following lemma.

Lemma 2.2. If $g$ satisfies $\left(g_{1}\right)$ and either $\left(g_{2}\right)$ or $\left(g_{4}\right)$, then the function $\boldsymbol{w} \equiv \phi$ and the functional $\widetilde{J}$ are even. 
Proof. Let $\mathbf{z}=(z,-z) \in Z$. First, let us verify that

$$
\langle\nabla J(-z+w(z), z+w(z)),(\varphi, \varphi)\rangle=0, \quad \forall \varphi \in H_{0}^{1}(\Omega)
$$

which, by uniqueness in (i) of Lemma 2.1, implies that $\mathbf{w}(\mathbf{z})=\mathbf{w}(-\mathbf{z})$. Indeed, observe that

$$
\begin{aligned}
& \langle\nabla J(-z+w(z), z+w(z)),(\varphi, \varphi)\rangle \\
& =\partial_{u} J(-z+w(z), z+w(z)) \varphi+\partial_{v} J(-z+w(z), z+w(z)) \varphi \\
& =\int_{\Omega} \nabla \varphi \cdot \nabla(z+w(z))+g(-z+w(z)) \varphi d \zeta+\int_{\Omega} \nabla(-z+w(z)) \cdot \nabla \varphi+g(z+w(z)) \varphi d \zeta \\
& =\int_{\Omega} \nabla \varphi \cdot \nabla(-z+w(z))+g(z+w(z)) \varphi d \zeta+\int_{\Omega} \nabla(z+w(z)) \cdot \nabla \varphi+g(-z+w(z)) \varphi d \zeta \\
& =\partial_{u} J(z+w(z),-z+w(z)) \varphi+\partial_{v} J(z+w(z),-z+w(z)) \varphi \\
& =\langle\nabla J(z+w(z),-z+w(z)),(\varphi, \varphi)\rangle=0, \quad \forall \varphi \in H_{0}^{1}(\Omega) .
\end{aligned}
$$

Hence, given $z \in H_{0}^{1}(\Omega)$,

$$
\begin{aligned}
\widetilde{J}(-\mathbf{z}) & =J(-z+w(-z), z+w(-z)) \\
& =J(-z+w(z), z+w(z)) \\
& =\int_{\Omega}\left(|\nabla w(z)|^{2}-|\nabla(-z)|^{2}+G(-z+w(z))+G(z+w(z))\right) d \zeta \\
& =J(z+w(z),-z+w(z)) \\
& =\widetilde{J}(\mathbf{z}) .
\end{aligned}
$$

Remark 1: Observe that from condition $\left(g_{1}\right)$ and Lemma 2.1, we conclude that the set of candidates to be solutions of (3) is contained in the graph $\{\mathbf{z}+\mathbf{w}(\mathbf{z}): \mathbf{z} \in Z\}$. From condition $\left(g_{0}\right)$ we have $\mathbf{w}(\mathbf{0})=\mathbf{0}$. Hence, combining these two facts, we observe that under $\left(g_{0}\right)-\left(g_{1}\right)$ the unique solution $(u, v)$ of $(3)$ with $u \equiv v$, i.e living in the set of fixed points of the action group, is the trivial one. Compare this with Theorem C.

\section{Proof of Theorem A}

Throughout this section we assume $g$ satisfies $\left(g_{0}\right),\left(g_{1}\right),\left(g_{2}\right)$ and $\left(g_{3}\right)$. To prove Theorem A we make use of the following version of the Symmetric Mountain Pass Theorem (see e. g. $[\mathrm{R}]$ ). We recall that if $E$ is a Banach space and $I \in C^{1}(E, \mathbb{R})$, a sequence $\left\{e_{n}\right\}$ in $E$ is a (PS)-sequence for the functional $I$, provided that

$$
\forall n \in \mathbb{N}, \quad\left|I\left(e_{n}\right)\right| \leq C \quad \text { and } \quad D I\left(e_{n}\right) \longrightarrow 0, n \rightarrow \infty .
$$

The functional $I$ is said to satisfy the (PS)-condition on $E$ if every (PS)-sequence in $E$ has a convergent subsequence.

Theorem 3.1. Let $E=E_{1} \oplus E_{2}$ be an infinite dimensional Banach space, where $E_{1}$ is a finite dimensional subspace. Let us assume $I \in C^{1}(E, \mathbb{R})$ is even, satisfies the Palais-Smale condition and $I(0)=0$. Assume, in addition, I satisfies: 
$\left(I_{1}\right)$ There exist positive constants $\alpha$ and $\rho$ such that $\left.I\right|_{\partial B_{\rho} \cap E_{2}} \geq \alpha$.

$\left(I_{2}\right)$ For each finite dimensional subspace $X \subset E$ there exists an $R=R(X)>0$ such that $\left.I\right|_{X \backslash B_{R}(0)} \leq 0$.

Then I possesses an unbounded sequence of critical values.

We apply Theorem 3.1 to the functional $-\widetilde{J}$. To this end, let $j \in \mathbb{N}$ such that $g^{\prime}(0)<\lambda_{j}$. We take $E_{1}:=\left\langle\left(\varphi_{1},-\varphi_{1}\right) \ldots,\left(\varphi_{j-1},-\varphi_{j-1}\right)\right\rangle \subset Z$ and $E_{2}=E_{1}^{\perp} \subset Z$.

Claim 1: Under assumptions $\left(g_{0}\right)-\left(g_{3}\right)$ functional $-\widetilde{J}$ satisfies $\left(I_{1}\right)$.

Proof. Let us consider the functional $F: H_{0}^{1}(\Omega) \longrightarrow \mathbb{R}$ defined as

$$
\begin{aligned}
F(z) & =-J(z,-z)=\int_{\Omega}\left(|\nabla z|^{2}-G(z)-G(-z)\right) d \zeta \\
& =\int_{\Omega}\left(\frac{1}{2}|\nabla z|^{2}-G(z)\right) d \zeta+\int_{\Omega}\left(\frac{1}{2}|\nabla(-z)|^{2}-G(-z)\right) d \zeta .
\end{aligned}
$$

Because of hypothesis $\left(g_{0}\right)$ and the variational characterization of $\lambda_{j}$ (see $[\mathrm{R}]$ or $[\mathrm{CV}]),\left.F\right|_{\left\langle\varphi_{1} \ldots, \varphi_{j-1}\right\rangle^{\perp}}$ has a strict local minimum at zero and there exist positive constants $\alpha$ and $\rho$ such that

$$
F(z) \geq \alpha \quad \forall z \in \partial B_{\rho} \cap\left\langle\varphi_{1} \ldots, \varphi_{j-1}\right\rangle^{\perp} \subset H_{0}^{1}(\Omega) .
$$

Hence, for each $\mathbf{z}=(z,-z) \in \partial B_{\sqrt{2} \rho} \cap E_{2} \subset Z$,

$$
-\widetilde{J}(\mathbf{z})=-\min _{w \in H_{0}^{1}(\Omega)} J(z+w,-z+w) \geq-J(z,-z)=F(z) \geq \alpha .
$$

Claim 2: Under assumptions $\left(g_{0}\right)-\left(g_{3}\right)$ the functional $-\widetilde{J}$ satisfies $\left(I_{2}\right)$.

Proof. Let $X$ be a finite dimensional subspace of $Z$. Then, there exists a constant $\gamma_{X}>0$ such that $\|z\|^{2} \leq \gamma_{X}\|z\|_{L^{2}}^{2}$ for all $\mathbf{z}=(z,-z) \in X$. Using hypothesis $\left(g_{3}\right)$ and integrating,

$$
G(t) \geq a|t|^{\mu}-b
$$

where $a>0$ and $b>0$ are constants. Since $\mu>2$, given any $\alpha>0$, there exists a constant $C_{\alpha}$ such that

$$
a|t|^{\mu}-b \geq \frac{\alpha}{2} t^{2}+C_{\alpha}
$$

(for this, simply consider $h(t):=a|t|^{\mu}-\frac{\alpha}{2} t^{2}-b$, which is bounded below and continuous). Thus,

$$
G(t) \geq \frac{\alpha}{2} t^{2}+C_{\alpha} \quad \forall t \in \mathbb{R} .
$$

Therefore, given $\mathbf{z}=(z,-z) \in X, \mathbf{w}(\mathbf{z})=(w(z), w(z))$,

$$
G(z+w(z))+G(-z+w(z)) \geq \frac{\alpha}{2}(z+w(z))^{2}+\frac{\alpha}{2}(-z+w(z))^{2}+2 C_{\alpha} .
$$

We then have

$$
\begin{aligned}
-\widetilde{J}(\mathbf{z}) & =\int_{\Omega}\left[|\nabla z|^{2}-|\nabla w(z)|^{2}-G(z+w(z))-G(-z+w(z))\right] d \zeta \\
& \leq \gamma_{X} \int_{\Omega} z^{2} d \zeta-\alpha \int_{\Omega} z^{2} d \zeta-\alpha \int_{\Omega}(w(z))^{2} d \zeta-2 \widehat{C}_{\alpha} \\
& \leq\left(\gamma_{X}-\alpha\right) \int_{\Omega} z^{2}-2 \widehat{C}_{\alpha} .
\end{aligned}
$$


Thus, taking $\alpha>\gamma_{X}$, we have that

$$
-\widetilde{J}(\mathbf{z}) \longrightarrow-\infty, \text { as }\|\mathbf{z}\| \rightarrow \infty, \mathbf{z} \in X .
$$

Since, $X$ is arbitrary we have verified $\left(I_{2}\right)$.

It remains to show that $\widetilde{J}$ satisfies the Palais-Smale condition.

Lemma 3.1. Under the assumptions $\left(g_{0}\right)-\left(g_{3}\right)$ the functional $\widetilde{J}$ satisfies the (PS)condition.

Proof. Observe that from (11) and (12), it suffices to verify that $J$ satisfies the Palais-Smale condition. Let $\left\{\left(u_{n}, v_{n}\right)\right\}_{n} \subset H_{0}^{1}(\Omega) \times H_{0}^{1}(\Omega)$ be a (PS)-sequence. We want to extract a strongly convergent subsequence. Due to the form of $D J$, the compactness on the Sobolev Embeddings and Vainberg's Lemma (see e.g. [MZ]), we just have to prove that $\left\{u_{n}\right\}_{n}$ and $\left\{v_{n}\right\}_{n}$ are bounded sequences in $H_{0}^{1}(\Omega)$.

Condition (16) implies that there exists a sequence $\left\{\varepsilon_{n}\right\}_{n}, \varepsilon_{n}>0$ and $\varepsilon_{n} \rightarrow 0^{+}$so that

$$
\left|D J\left(u_{n}, v_{n}\right)[\phi, \psi]\right| \leq \varepsilon_{n}(\|\phi\|+\|\psi\|), \quad \forall \phi, \psi \in H_{0}^{1}(\Omega) .
$$

We take as test functions $\phi=\frac{1}{2} u_{n}$ and $\psi=\frac{1}{2} v_{n}$ to get

$$
\begin{aligned}
C & +\frac{\varepsilon_{n}}{2}\left(\left\|u_{n}\right\|+\left\|v_{n}\right\|\right) \\
\geq & \frac{1}{2} D J\left(u_{n}, v_{n}\right)\left[u_{n}, v_{n}\right]-J\left(u_{n}, v_{n}\right) \\
= & \int_{\Omega}\left\{-G\left(v_{n}\right)-G\left(u_{n}\right)\right\}+\frac{1}{2} \int_{\Omega}\left\{g\left(u_{n}\right) u_{n}+g\left(v_{n}\right) v_{n}\right\} \\
\geq & \frac{1}{2} \int_{\Omega}\left\{g\left(v_{n}\right) v_{n}-\mu G\left(v_{n}\right)\right\}+\frac{1}{2} \int_{\Omega}\left\{g\left(u_{n}\right)\left(u_{n}\right)-\mu G\left(u_{n}\right)\right\} \\
& \quad+\left(\frac{\mu}{2}-1\right) \int_{\Omega}\left\{G\left(v_{n}\right)+G\left(u_{n}\right)\right\} .
\end{aligned}
$$

So, changing the constant $C$ if necessary, we find by $\left(g_{3}\right)$ that

$$
\int_{\Omega} G\left(u_{n}\right)+G\left(v_{n}\right) \leq C\left[1+\varepsilon_{n}\left(\left\|u_{n}\right\|+\left\|v_{n}\right\|\right)\right] .
$$

Since $\left\{J\left(u_{n}, v_{n}\right)\right\}_{n}$ is bounded, we can choose a large positive constant $C$ such that

$$
\left|\int_{\Omega} \nabla u_{n} \cdot \nabla v_{n}+\int_{\Omega} G\left(u_{n}\right)+G\left(v_{n}\right)\right| \leq C
$$

Because of hypothesis $\left(g_{3}\right),|G(t)|-G(t)=0$, for every $|t| \geq R$, so it is a bounded function. Thus, we get from (18) and (19) that

$$
\begin{aligned}
\left|\int_{\Omega} \nabla u_{n} \cdot \nabla v_{n}\right| & \leq \int_{\Omega}\left|G\left(u_{n}\right)\right|+\left|G\left(v_{n}\right)\right|+C \\
& \leq \int_{\Omega} G\left(u_{n}\right)+G\left(v_{n}\right)+C \\
& \leq C\left[1+\varepsilon_{n}\left(\left\|u_{n}\right\|+\left\|u_{n}\right\|\right)\right] .
\end{aligned}
$$


From (17), testing against $[\phi, \psi]=\left[u_{n}, v_{n}\right]$, we obtain

$$
\left|2 \int_{\Omega} \nabla u_{n} \cdot \nabla v_{n}+\int_{\Omega} g\left(u_{n}\right) u_{n}+g\left(v_{n}\right) v_{n}\right| \leq \varepsilon_{n}\left(\left\|u_{n}\right\|+\left\|u_{n}\right\|\right) .
$$

So, by (20) we obtain

$$
\int_{\Omega} g\left(u_{n}\right) u_{n}+g\left(v_{n}\right) v_{n} \leq C\left[1+\varepsilon_{n}\left(\left\|u_{n}\right\|+\left\|u_{n}\right\|\right)\right] .
$$

On the other hand, using again (17) and testing against $[\phi, \psi]=\left[0, u_{n}\right]$, we have

$$
\left.\left|\int_{\Omega}\right| \nabla u_{n}\right|^{2}+g\left(v_{n}\right) u_{n} \mid \leq \varepsilon_{n}\left\|u_{n}\right\|
$$

Now let us estimate the second term in left-hand side of inequality (22). Using Hölder inequality we have

$$
\left|\int_{\Omega} g\left(v_{n}\right) u_{n}\right| \leq\left(\int_{\Omega}\left|g\left(v_{n}\right)\right|^{1+\frac{1}{p}}\right)^{\frac{p}{1+p}}\left(\int_{\Omega}\left|u_{n}\right|^{1+p}\right)^{\frac{1}{1+p}}
$$

Now note that for suitable positive constants $c, d_{1}, d_{2}$,

$$
|g(t)|^{1+\frac{1}{p}} \leq c|g(t)||t|+d_{1} \leq c g(t)+d_{2} .
$$

Indeed, the first inequality in (24) follows from hypothesis $\left(g_{2}\right)$, since

$$
|g(t)|^{\frac{1}{p}} \leq C|t|+d:
$$

- for $|t| \geq 1$

$$
\begin{aligned}
|g(t)|^{1+\frac{1}{\bar{p}}} & \leq C|g(t)||t|+d|g(t)| \\
& \leq C|g(t)||t|+d|g(t)||t| .
\end{aligned}
$$

- for $|t| \leq 1$ we see that $|g(t)|$ is simply bounded. So the first inequality in (24) holds. As for the second inequality in (24), we write

$$
|g(t)||t|=g(t) \cdot t+|g(t)||t|-g(t) \cdot t,
$$

and observe that, because of $\left(g_{3}\right),|g(t)||t|-g(t) \cdot t=0$, for $|t| \geq R$. So this difference remains bounded in $\mathbb{R}$ and the inequality holds.

From (21), (23) and (24), we get that

$$
\begin{aligned}
\left|\int_{\Omega} g\left(v_{n}\right) u_{n}\right| & \leq\left(c \int_{\Omega} g\left(v_{n}\right) v_{n}+d_{2}\right)^{\frac{p}{1+p}}\left\|u_{n}\right\|_{L^{1+p}} \\
& \leq\left(C\left[1+\varepsilon_{n}\left(\left\|u_{n}\right\|+\left\|v_{n}\right\|\right)\right]\right)^{\frac{p}{1+p}}\left\|u_{n}\right\| .
\end{aligned}
$$

Then, by (22),

$$
\int_{\Omega}\left|\nabla u_{n}\right|^{2} \leq \varepsilon_{n}\left\|u_{n}\right\|+\left(C\left[1+\varepsilon_{n}\left(\left\|u_{n}\right\|+\left\|v_{n}\right\|\right)\right]\right)^{\frac{p}{1+p}}\left\|u_{n}\right\| .
$$


In a similar fashion, taking $[\phi, \psi]=\left[v_{n}, 0\right]$ in $(17)$, we get the analogous estimate

$$
\int_{\Omega}\left|\nabla v_{n}\right|^{2} \leq \varepsilon_{n}\left\|v_{n}\right\|+\left(C\left[1+\varepsilon_{n}\left(\left\|u_{n}\right\|+\left\|v_{n}\right\|\right)\right]\right)^{\frac{p}{1+p}}\left\|v_{n}\right\| .
$$

Joining these two estimates we obtain

$$
\left\|u_{n}\right\|^{2}+\left\|v_{n}\right\|^{2} \leq \varepsilon_{n}\left(\left\|u_{n}\right\|+\left\|v_{n}\right\|\right)+C\left(\left\|u_{n}\right\|+\left\|v_{n}\right\|\right)^{\frac{2 p+1}{1+p}}+K .
$$

Since $\frac{2 p+1}{1+p}<2$, the sequence $\left\{\left(u_{n}, v_{n}\right)\right\}_{n}$ is bounded in $H$ and the proof of the lemma is complete.

\section{Proof of Theorem B}

Throughout this section we assume that $g$ satisfies $\left(g_{0}\right),\left(g_{1}\right)$ and $\left(g_{4}\right)$. To prove Theorem B we make use of the following version of the Symmetric Mountain Pass Theorem (see e.g. [AR], [BBF], and $[\mathrm{S}]$ ).

Theorem 4.1. Let $E=E_{1} \oplus E_{2}$ be a real Banach space, where $E_{1}$ is a finite dimensional subspace. Let $X \subset E$ be a finite dimensional subspace of $E$ such that $\operatorname{dim} E_{1}<\operatorname{dim} X$. Suppose that $I \in C^{1}(E, \mathbb{R})$ is an even functional, satisfying $I(\boldsymbol{O})=0$ and

$\left(I_{1}^{\prime}\right)$ There exists a positive constant $\rho$ such that $\left.I\right|_{\partial B_{\rho} \cap E_{2}} \geq 0$.

$\left(I_{2}^{\prime}\right)$ There exists $M>0$ such that $\max _{z \in X} I(z)<M$.

If I satisfies the Palais-Smale condition at level $c$, for every $c \in[0, M]$, then $I$ possesses (at least) $\operatorname{dim} X-\operatorname{dim} E_{1}$ pairs of nontrivial critical points.

As in Section 3, we take $E_{1}:=\left\langle\left(\varphi_{1},-\varphi_{1}\right) \ldots,\left(\varphi_{j-1},-\varphi_{j-1}\right)\right\rangle$ and $E_{2}=E_{1}^{\perp}$. As we proved in the previous section, the fact that $-\widetilde{J}$ satisfies $\left(I_{1}^{\prime}\right)$ comes from hypothesis $\left(g_{0}\right)$ and the variational characterization of the eigenvalues, i.e. the local structure of the functional around zero in this case is similar to that of the superlinear setting.

Claim: Under hypotheses $\left(g_{0}\right),\left(g_{1}\right)$ and $\left(g_{4}\right)$, the functional $-\widetilde{J}$ satisfies $\left(I_{2}^{\prime}\right)$.

Proof. Let us take $X=\left\langle\left(\varphi_{1},-\varphi_{1}\right) \ldots,\left(\varphi_{k},-\varphi_{k}\right)\right\rangle$. Since $g^{\prime}(\infty)>\lambda_{k}$, taking a number $\alpha \in\left(\lambda_{k}, g^{\prime}(\infty)\right)$ it follows that

$$
G(t)>\frac{\alpha}{2} t^{2}+C_{\alpha} \quad \forall t \in \mathbb{R} .
$$

The remaining of this proof is very similar to the proof of Claim 2 in Section 3 by simply using the inequality

$$
\|x\|^{2} \leq \lambda_{k} \int_{\Omega} x^{2} \quad \forall x \in\left\langle\varphi_{1}, \ldots, \varphi_{k}\right\rangle .
$$

From this, given $\mathbf{z}=(z,-z) \in X$,

$$
-\widetilde{J}(\mathbf{z}) \leq\left(\lambda_{k}-\alpha\right)\|z\|_{L^{2}}^{2}+\widetilde{C}_{\alpha} \longrightarrow-\infty \text { as }\|\mathbf{z}\| \rightarrow \infty, \mathbf{z} \in X .
$$

It remains to show that $\widetilde{J}$ satisfies the Palais-Smale condition. In this case, we follow the ideas of the corresponding proof for the problem with one equation and asymptotic (nonresonant) nonlinearities, although our proof requires a bit more of technicalities. 
Lemma 4.1. Under assumptions $\left(g_{0}\right),\left(g_{1}\right)$ and $\left(g_{4}\right)$ the functional $\widetilde{J}$ satisfies the (PS)-condition.

Proof. As before, from (11) and (12), it suffices to verify that $J$ satisfies the PalaisSmale condition. We take a (PS)-sequence $\left\{\left(u_{n}, v_{n}\right)\right\}_{n}$ in $H_{0}^{1}(\Omega) \times H_{0}^{1}(\Omega)$ and again it is sufficient to prove that this sequence is bounded. In this case, we argue by contradiction. Let us assume that $\left\{\left\|\left(u_{n}, v_{n}\right)\right\|\right\}_{n}$ is not bounded. Passing to a subsequence, denoted the same for simplicity of notation, we can say that either $\left\|u_{n}\right\| \rightarrow \infty$ or $\left\|v_{n}\right\| \rightarrow \infty$. We claim that

(I) if $\left\|u_{n}\right\| \rightarrow \infty$, then there exists a subsequence $\left\|v_{n_{k}}\right\| \rightarrow \infty$, and

(II) if $\left\|v_{n}\right\| \rightarrow \infty$, then there exists a subsequence $\left\|u_{n_{k}}\right\| \rightarrow \infty$.

Indeed, let us prove $(I)$ arguing by contradiction. If $\left\|u_{n}\right\| \rightarrow \infty$ and $\left\|v_{n}\right\| \leq C$, then, passing to a subsequence we have that

$$
\begin{array}{lll}
v_{n} \rightarrow v, \quad \text { in } H_{0}^{1}(\Omega) & \frac{u_{n}}{\left\|u_{n}\right\|} \rightarrow \bar{u}, \quad \text { in } H_{0}^{1}(\Omega) \\
v_{n} \rightarrow v, \quad \text { in } L^{r}(\Omega) & \frac{u_{n}}{\left\|u_{n}\right\|} \rightarrow \bar{u}, \text { in } L^{r}(\Omega), \text { for } r \in\left[1, \frac{2 N}{N-2}\right) .
\end{array}
$$

There exists a sequence $\left\{\varepsilon_{n}\right\}_{n}, \varepsilon_{n}>0$ and $\varepsilon_{n} \rightarrow 0^{+}$so that

$$
\left|D J\left(u_{n}, v_{n}\right)[\phi, \psi]\right| \leq \varepsilon_{n}(\|\phi\|+\|\psi\|), \quad \forall \phi, \psi \in H_{0}^{1}(\Omega) .
$$

Testing $\partial_{v} J\left(u_{n}, v_{n}\right)$ against $\frac{u_{n}}{\left\|u_{n}\right\|}$ and using (25) we get that

$$
\left|\left\|u_{n}\right\|+\int_{\Omega} g\left(v_{n}\right) \frac{u_{n}}{\left\|u_{n}\right\|}\right| \leq \varepsilon_{n} .
$$

From $\left(g_{4}\right),|g(t)| \leq C(1+|t|)$ for all $t \in \mathbb{R}$. Using Vainberg's Lemma (see [MZ]) we have that

$$
\int_{\Omega} g\left(v_{n}\right) \frac{u_{n}}{\left\|u_{n}\right\|} \longrightarrow \int_{\Omega} g(v) \bar{u}
$$

and so we get

$$
\left\|u_{n}\right\| \longrightarrow-\int_{\Omega} g(v) \bar{u}, \text { as } n \rightarrow \infty .
$$

This contradicts our initial assumption. We proceed in an analogue way to prove $(I I)$ and therefore the claim is proved.

Now, using the claim, and passing to a subsequence, we can assume without loss of generality that:

$$
\left\|u_{n}\right\| \rightarrow \infty \quad \text { and } \quad\left\|v_{n}\right\| \rightarrow \infty .
$$

Hence, there exist $u, v \in H_{0}^{1}(\Omega)$ such that

$$
\begin{array}{lll}
\frac{u_{n}}{\left\|u_{n}\right\|} \rightarrow \bar{u}, \quad \text { in } H_{0}^{1}(\Omega) & \frac{v_{n}}{\left\|v_{n}\right\|} \rightarrow \bar{v}, \text { in } H_{0}^{1}(\Omega) \\
\frac{u_{n}}{\left\|u_{n}\right\|} \rightarrow \bar{u}, \quad \text { in } L^{r}(\Omega) & \frac{v_{n}}{\left\|v_{n}\right\|} \rightarrow \bar{v}, \quad \text { in } L^{r}(\Omega), \text { for } r \in\left[1, \frac{2 N}{N-2}\right) .
\end{array}
$$

We claim that $\left\{\left\|u_{n}\right\|\right\}_{n}$ and $\left\{\left\|v_{n}\right\|\right\}_{n}$ go to infinity at the same rate. More precisely, we claim that

$$
\lim _{n \rightarrow \infty} \frac{\left\|u_{n}\right\|}{\left\|v_{n}\right\|}=1
$$


To prove this claim, we first test $\partial_{u} J\left(u_{n}, v_{n}\right)$ against $\frac{v_{n}}{\left\|v_{n}\right\|}$ and then divide by $\left\|u_{n}\right\|$ to get

$$
\left|\frac{\left\|v_{n}\right\|}{\left\|u_{n}\right\|}+\int_{\Omega} \frac{g\left(u_{n}\right)}{\left\|u_{n}\right\|} \cdot \frac{v_{n}}{\left\|v_{n}\right\|}\right| \leq \frac{\varepsilon_{n}}{\left\|u_{n}\right\|} .
$$

Assumption $\left(g_{4}\right)$ implies that $g(t)=g^{\prime}(\infty) t+\gamma(t)$, where $\gamma(t)=o(t)$, as $|t| \rightarrow \infty$. Then,

$$
\int_{\Omega} \frac{g\left(u_{n}\right)}{\left\|u_{n}\right\|} \frac{v_{n}}{\left\|v_{n}\right\|}=g^{\prime}(\infty) \int_{\Omega} \frac{v_{n}}{\left\|v_{n}\right\|} \frac{u_{n}}{\left\|u_{n}\right\|}+\int_{\Omega} \gamma\left(u_{n}\right) \frac{v_{n}}{\left\|v_{n}\right\|\left\|u_{n}\right\|} .
$$

Now we show that

$$
\int_{\Omega} \gamma\left(u_{n}\right) \frac{v_{n}}{\left\|v_{n}\right\|\left\|u_{n}\right\|} \longrightarrow 0
$$

Indeed, just observe that given $\varepsilon>0$ arbitrary, there exists $T \geq 0$ such that

$$
\left|\frac{\gamma(t)}{t}\right|<\varepsilon, \text { for }|t| \geq T \text {. }
$$

On the other hand, $\gamma(t)=g(t)-g^{\prime}(\infty) t$ is continuous in $[-T, T]$ and so it is bounded in $[-T, T]$. Thus, it follows that

$$
\begin{aligned}
\int_{\Omega}\left|\gamma\left(u_{n}\right) \frac{v_{n}}{\left\|v_{n}\right\|\left\|u_{n}\right\|}\right| & \leq \int_{\left\{\left|u_{n}\right|>T\right\}}+\int_{\left\{\left|u_{n}\right| \leq T\right\}} \\
& \leq \varepsilon \int_{\Omega}\left|\frac{u_{n}}{\left\|u_{n}\right\|} \frac{v_{n}}{\left\|v_{n}\right\|}\right|+\frac{C_{T}}{\left\|u_{n}\right\|} \int_{\Omega}\left|\frac{v_{n}}{\left\|v_{n}\right\|}\right| \\
& \leq C \varepsilon+\frac{C_{T}}{\left\|u_{n}\right\|} C \\
& \leq 2 C \varepsilon, \text { for } n \text { large enough. }
\end{aligned}
$$

Hence, we can take the limit in (28) to get

$$
\int_{\Omega} \frac{g\left(u_{n}\right)}{\left\|u_{n}\right\|} \frac{v_{n}}{\left\|v_{n}\right\|} \longrightarrow \int_{\Omega} g^{\prime}(\infty) \bar{u} \bar{v} .
$$

This and (27) give

$$
\frac{\left\|v_{n}\right\|}{\left\|u_{n}\right\|} \longrightarrow-\int_{\Omega} g^{\prime}(\infty) \bar{u} \bar{v}
$$

Arguing in a similar fashion, but now testing $\partial_{v} J\left(u_{n}, v_{n}\right)$ against $\frac{u_{n}}{\left\|u_{n}\right\|}$, we also obtain

$$
\frac{\left\|u_{n}\right\|}{\left\|v_{n}\right\|} \longrightarrow-\int_{\Omega} g^{\prime}(\infty) \bar{u} \bar{v}
$$

which together with (29) implies that actually $\int_{\Omega} g^{\prime}(\infty) \bar{u} \bar{v}=-1$ and therefore the claim is proved.

Let us now take $\phi \in H_{0}^{1}(\Omega)$. Using (25) we have that

$$
\left|\int_{\Omega} \nabla \phi \cdot \nabla\left(\frac{v_{n}}{\left\|v_{n}\right\|}\right)+\frac{g\left(u_{n}\right)}{\left\|v_{n}\right\|} \phi\right| \longrightarrow 0 .
$$


Due to the weak convergence of $\frac{v_{n}}{\left\|v_{n}\right\|}$ to $\bar{v}$, we know that

$$
\int_{\Omega} \nabla \phi \cdot \nabla\left(\frac{v_{n}}{\left\|v_{n}\right\|}\right) \longrightarrow \int_{\Omega} \nabla \phi \cdot \nabla \bar{v}
$$

On the other hand, (26) implies that

$$
\int_{\Omega} \frac{g\left(u_{n}\right)}{\left\|v_{n}\right\|} \phi \longrightarrow \int_{\Omega} g^{\prime}(\infty) \bar{u} \phi .
$$

To see why this is true, it is enough to notice that

$$
\int_{\Omega} \frac{g\left(u_{n}\right)}{\left\|v_{n}\right\|} \phi=\int_{\Omega} \frac{g\left(u_{n}\right)}{\left\|u_{n}\right\|} \cdot \frac{\left\|u_{n}\right\|}{\left\|v_{n}\right\|} \phi=\frac{\left\|u_{n}\right\|}{\left\|v_{n}\right\|} \int_{\Omega} \frac{g^{\prime}(\infty) u_{n}+\gamma\left(u_{n}\right)}{\left\|u_{n}\right\|} \phi
$$

and arguing as above, it can be proved that $\int_{\Omega} \frac{\gamma\left(u_{n}\right)}{\left\|u_{n}\right\|} \phi \longrightarrow 0$.

From (31), (32) and (33), we have proven that

$$
\forall \phi \in H_{0}^{1}(\Omega): \int_{\Omega} \nabla \bar{v} \cdot \nabla \phi+g^{\prime}(\infty) \bar{u} \phi=0 .
$$

Using (29) and reasoning analogously, we also get that

$$
\forall \phi \in H_{0}^{1}(\Omega): \int_{\Omega} \nabla \bar{u} \cdot \nabla \phi+g^{\prime}(\infty) \bar{v} \phi=0 .
$$

From relations (34) and (35), testing both integrals against $\phi=\bar{v}+\bar{u}$ we obtain

$$
\int_{\Omega}|\nabla(\bar{u}+\bar{v})|^{2}=-g^{\prime}(\infty) \int_{\Omega}(\bar{v}+\bar{u})^{2} .
$$

Since $g^{\prime}(\infty)>0, \bar{v}=-\bar{u}$. Replacing this in any of the relations (34) or (35) we get that $\bar{u}=-\bar{v} \in H_{0}^{1}(\Omega)$ is a weak solution, and actually a classical one, to the problem

$$
\left\{\begin{aligned}
-\Delta u & =g^{\prime}(\infty) u \text { in } \Omega \\
u & =0 \text { on } \partial \Omega .
\end{aligned}\right.
$$

This, as well as (29) and (30), imply that $g^{\prime}(\infty)=\lambda_{j}$ for some $j \in \mathbb{N}$. This contradicts hypothesis $\left(g_{4}\right)$. Hence, a contradiction is reached assuming that $\left\{\left\|\left(u_{n}, v_{n}\right)\right\|\right\}_{n}$ is unbounded, and the conclusion of the lemma follows.

\section{Proof of Theorem C}

Assume condition $\left(g_{5}\right)$. Let us assume $(u, v)$ is a solution of (3). Multiply the first equation in (3) by $u-v$, and then multiply the second equation by $u-v$. Taking the difference of both results, we get

$$
\int_{\Omega}|\nabla(u-v)|^{2}+(g(v)-g(u))(u-v)=0
$$

or, equivalently,

$$
\int_{\Omega}|\nabla(u-v)|^{2}=\int_{\Omega}(g(u)-g(v))(u-v) .
$$


Because of Mean Value Theorem and $\left(g_{5}\right)$, we have that

$$
\int_{\Omega}|\nabla(u-v)|^{2} \leq\left(\lambda_{1}-\epsilon\right) \int_{\Omega}(u-v)^{2},
$$

for some small $\epsilon>0$. From Poincarè's Inequality we conclude that $u \equiv v$.

\section{References}

[A] R. A. Adams, Sobolev Spaces, New York, Academic Press, 1975.

[AR] A. Ambrosetti, P.H. Rabinowitz, Dual variational methods in critical point theory and applications, J. Funct. Anal. 14 (1973) 349-381.

[BBF] P. Bartolo, V. Benci, D. Fortunato, Abstract critical point theorems and applications to some nonlinear problems with "strong" resonance at infinity, Nonlinear Anal. TMA 7 (9) (1983) 981-1012.

[C] A. Castro, Reduction methods via minimax, Lecture Notes in Mathematics 957, Differential Equations, Springer Berlin-New York, 1982, pp.1-20.

[CL] A. Castro, A. Lazer, Critical point theory and the number of solutions of a nonlinear Dirichlet problem, Ann. Mat. Pura Appl., (4) 120 (1979), 113-137.

[CV] J. Cossio and C. Vélez, Soluciones no triviales para un problema de Dirichlet asintóticamente lineal, Rev. Colombiana Mat. Vol 37, 2003, 25-36.

[DF] D. G. De Figueiredo and P. L. Felmer, On Supercuadratic Elliptic Systems, Trans. of AMS, Volume 343, Number 1, 1994, pp. 99-116.

[FSX] M. Furtado, E. Silva, M. Xavier, Multiplicity and Concentration of Solutions for Elliptic Systems with Vanishing Potentials, J. Diff. Eq. 249 (2010), pp. 23772396.

[GT] D. Gilbart, N.S. Trudinger, Elliptic partial differential equations of second order, Springer Verlag. Berlin 1977.

[MZ] D. Mitrovic and D. Zubrinic, Fundamentals of applied functional analysis, Pitman Monographs and surveys in pure and applied Mathematics, 91. Addison Wesley. Longman Inc., 1998

[R] P. H. Rabinowitz, Minimax methods in Critical Point theory with applications to Differential Equations, Regional Conference Series in Mathematics, number 65. AMS, Providence, R.I., 1986.

[S] E.A.B. Silva, Critical point theorems and applications to differential equations, Ph.D. Thesis, University of Wisconsin-Madison, 1988.

Oscar Agudelo

Departamento de Ingeniería Matemática 
Universidad de Chile

Casilla 170 Correo 3

Santiago de Chile

E-mail address:

oagudelo@dim.uchile.cl

Bernhard Ruf

Dipartimento di Matematica

Università degli Studi di Milano

20133 Milano, Italia

E-mail address:

bernhard.ruf@unimi.it

Carlos Vélez

Escuela de Matemáticas

Universidad Nacional de Colombia, Sede Medellín

Apartado Aéreo 3840, Medellín, Colombia

E-mail addresses:

cauvelez@unal.edu.co 